Original Article

Journal of Epilepsy Research pISSN 2233-6249 / eISSN 2233-6257

Received December 14, 2018

Revised May 10, 2020

Accepted June 12, 2020

Corresponding author:

Rayees Ahmad Tarray, DM

Department of Neurology, SKIMS, Srinagar

190011, India

Tel. $+91-9622755432$

E-mail; rayeestarray@gmail.com

\section{Comparative Efficacy of IV Phenytoin, IV Valproate, and IV Levetiracetam in Childhood Status Epilepticus}

\author{
Mudasir Nazir, MD ${ }^{1}$, Rayees Ahmad Tarray, DM², Ravouf Asimi, DM², \\ Wajid Ali Syed, MD²
}

Departments of ${ }^{1}$ Paediatrics and ${ }^{2}$ Neurology, SKIMS, Srinagar, India

\begin{abstract}
Background and Purpose: Status epilepticus (SE) is a common pediatric neurological emergency that requires immediate and vigorous management. Currently, phenytoin is the most common agent used in the setting of SE following benzodiazepine for further seizure prevention. Other drugs recently introduced for management of SE are valproic acid and levetiracetam.
\end{abstract}

Methods: This prospective randomized study included 150 pediatric patients admitted as SE. Patients were randomized into three equal groups (50 each) to receive one of the three anticonvulsants in addition to standard treatment. Patients were monitored in hospital regarding their vitals, time to regain consciousness, and seizure recurrence.

Results: At 24 hours seizures were controlled in 44 patients (88\%) in phenytoin group, 39 patients (78\%) in levetiracetam (LEV) group and 46 patients $(92 \%)$ in valproate (VAL) group $(p=0.115)$. The mean time to regain consciousness in phenytoin, LEV and VAL groups was 122.3 $\pm 45.4,120.8 \pm 42.8$, and $75.0 \pm 30.7$ minutes (mean \pm standard deviation) respectively. Patients in VAL group regained consciousness earlier than both phenytoin and LEV group patients $(p<0.0001)$. At 3 months follow-up, seven $(14.28 \%)$ out of 49 patients in phenytoin group, $14(28.57 \%)$ out of 49 in LEV group and two (4\%) out of 50 patients in VAL group had a seizure recurrence $(p=0.0032)$.

Conclusions: In our study we found that both IV LEV and IV VAL safe and efficacious. The primary outcome, seizure recurrence at 24 hours, did not show a statistically significant difference in three groups $(p>0.05)$. Also, seizure recurrence at 1 week did not reach a statistically significant difference. However, time to regain consciousness and seizure recurrence at 3 months was significantly less in VAL group $(p<0.05)$. (2020;10:69-73)

Key words: Status epilepticus, Child, Phenytoin, Valproic acid, Leveteracetam

\section{Introduction}

Seizures are the most common pediatric neurologic disorder, with $4 \%$ to $10 \%$ of children suffering at least one seizure in the first 16 years of life. ${ }^{1}$ The incidence is highest in children younger than 3 years of age, with a decreasing frequency in older children. ${ }^{2}$ Status epilepticus (SE) is a common pediatric neurological emergency that requires immediate and vigorous management and at times poses a therapeutic challenge to the treating physician. If not managed promptly, it may result in significant neuromorbidity and mortality. ${ }^{3,4}$ In 1981, the International League Against Epilepsy defined SE as a single seizure or recurrent seizures lasting for more than 30 minutes during which consciousness is not regained. ${ }^{5}$ Practically, most seiz- ures resolve within few minutes, so seizures persisting longer than 5 minutes should probably be treated as SE. It is estimated that 1.3\% to $16 \%$ of all patients with epilepsy will develop SE at some point in their lives. ${ }^{4}$ Approximately $70 \%$ of SE occurs in children less than 1 year and $75 \%$ in less than 3 years of age ${ }^{6}$ and the first episode most commonly occurs around 2.5 years after initial diagnosis. ${ }^{7}$

The correct management strategy involves initial stabilization of airways, breathing and circulation, prompt control of seizures, evaluation and treatment of the underlying etiology. ${ }^{8-10}$ The standard protocol for treatment of pediatric SE involves use of a benzodiazepine first followed by a long acting drug like phenytoin. Among long acting agents, phenytoin is currently the most common agent used in the setting of acute seizure prevention in children. Phenobarbital, the 
long-acting potent antiepileptic drug, is used when benzodiazepines and phenytoin are ineffective to control seizures. Other drugs recently introduced for management of SE are valproic acid and levetiracetam. Many studies have reported safe and efficacious use of levetiracetam ${ }^{11-16}$ and valproate ${ }^{17-19}$ in SE. The present study has been devised to compare efficacies of phenytoin, levetiracetam and valproate as second-line SE treatment in children.

\section{Methods}

This prospective randomized study was conducted in the department of Pediatrics at Sher-i-Kashmir Institute of Medical Sciences, Srinagar during 2012-2014. Pediatric patients in the age group of 2 months to 16 years who presented as focal motor status or generalized convulsive status to the emergency department of pediatrics were included in the study. All the patients were informed about the purpose of the study and written informed consent was obtained from them. After taking approval from the hospital ethics committee, patients were randomized into three different groups. Randomization was done using a computer derived random-number sequence. One hundred fifty pediatric patients of either sex in the age group of 2 months to 16 years were enrolled in the study. The patients with following characteristics were excluded: 1) age below 1 month, 2) children already receiving antiepileptic drugs, 3) children with evi-

Table 1. Baseline characteristics of three groups

\begin{tabular}{|c|c|c|c|}
\hline \multirow[b]{2}{*}{ Baseline feature } & \multicolumn{3}{|c|}{ Study group } \\
\hline & $\begin{array}{l}\text { Phenytoin } \\
(n=50)\end{array}$ & $\begin{array}{l}\text { Levetiracetam } \\
\qquad(n=50)\end{array}$ & $\begin{array}{l}\text { Valproate } \\
\qquad(n=50)\end{array}$ \\
\hline Age (years) & $5.17 \pm 3.71$ & $4.98 \pm 4.14$ & $4.45 \pm 3.68$ \\
\hline \multicolumn{4}{|l|}{ Gender } \\
\hline Male & 35 & 36 & 33 \\
\hline Female & 15 & 14 & 17 \\
\hline Weight (kg) & $14.89 \pm 7.8$ & $14.75 \pm 8.88$ & $13.61 \pm 8.74$ \\
\hline Height (cm) & $86.48 \pm 54.88$ & $84.62 \pm 49.84$ & $86.48 \pm 50.98$ \\
\hline $\mathrm{HC}(\mathrm{cm})$ & $45.76 \pm 5.56$ & $46.16 \pm 5.66$ & $45.80 \pm 5.57$ \\
\hline BMI $\left(k g / m^{2}\right)$ & $14.11 \pm 1.77$ & $14.23 \pm 2.33$ & $14.40 \pm 1.66$ \\
\hline \multicolumn{4}{|l|}{ Etiology } \\
\hline Idiopathic & 39 & 38 & 39 \\
\hline Congenital/perinatal & 5 & 4 & 4 \\
\hline Febrile status & 3 & 4 & 3 \\
\hline Infections & 2 & 2 & 3 \\
\hline Vascular & 1 & 2 & 1 \\
\hline
\end{tabular}

Values are presented as mean \pm standard deviation or number. dence of meningitis or head trauma, and 4) known hypersensitivity to drugs in study.

After initial stabilization and assessment, patients were randomly assigned to three groups of: 1) phenytoin group - received IV phenytoin loading dose at $20 \mathrm{mg} / \mathrm{kg}$ diluted in NS at a rate $<1 \mathrm{mg} / \mathrm{kg} / \mathrm{min}$ followed by maintenance dose of $5 \mathrm{mg} / \mathrm{kg} /$ day in two divided doses. 2) levetiracetam group - received IV levetiracetam loading dose 25 $\mathrm{mg} / \mathrm{kg}$ at $3 \mathrm{mg} / \mathrm{kg} / \mathrm{min}$ followed by maintenance $25 \mathrm{mg} / \mathrm{kg} /$ day divided 12 hourly. And 3) valproate group - received IV valproate loading $25 \mathrm{mg} / \mathrm{kg}$ at $3 \mathrm{mg} / \mathrm{kg} / \mathrm{h}$ followed by maintenance $20 \mathrm{mg} / \mathrm{kg} / \mathrm{day}$ in divided doses 12 hourly.

The primary outcome measure in our study was the cessation of seizure activity for 24 hours. Secondary outcome measures were effect on vital parameters, time to regain consciousness, adverse reactions and seizure recurrence at 1 week and 3 months. The observed data was entered in the computer to analyze with Excel (Microsoft, Redmond, WA, USA) and SPSS version 15 for windows (IBM, New York, NY, USA). The primary outcome measure is presented as mean and standard deviation (SD) and statistically significant difference was evaluated using one-way analysis of variance. Statistically significant difference of qualitative variables among three groups was evaluated using chi square/Fischers exact test. A $p$-value of $<0.05$ was considered as significant and a $p$-value less

Table 2. Monitoring of vital parameters

\begin{tabular}{lccc}
\hline Parameter & \multicolumn{3}{c}{ Study group } \\
\cline { 2 - 4 } & $\begin{array}{c}\text { Phenytoin } \\
(n=50)\end{array}$ & $\begin{array}{c}\text { Levetiracetam } \\
(n=50)\end{array}$ & $\begin{array}{c}\text { Valproate } \\
(n=50)\end{array}$ \\
\hline HR & $125 \pm 23$ & $126 \pm 24$ & $127 \pm 19$ \\
Basal & $112 \pm 20$ & $115 \pm 21$ & $118 \pm 17$ \\
30 minutes & $109 \pm 19$ & $111 \pm 2$ & $115 \pm 17$ \\
60 minutes & & & \\
SBP & $93 \pm 10$ & $91 \pm 10$ & $95 \pm 11$ \\
Basal & $93 \pm 10$ & $90 \pm 10$ & $94 \pm 11$ \\
30 minutes & $89 \pm 9$ & $87 \pm 9$ & $91 \pm 10$ \\
60 minutes & & & \\
RR & $28 \pm 5$ & $29 \pm 6$ & $29 \pm 4$ \\
Basal & $25 \pm 5$ & $26 \pm 6$ & $26 \pm 4$ \\
30 minutes & $25 \pm 5$ & $26 \pm 5$ & $25 \pm 4$ \\
60 minutes & & & \\
SpO & $95 \pm 3$ & $95 \pm 3$ & $94 \pm 3$ \\
Basal & $98 \pm 1$ & $99 \pm 1$ & $98 \pm 1$ \\
60 minutes & & &
\end{tabular}

$H R$, heart rate; SBP, systolic blood pressure; RR, respiratory rate. 
than $0.001(p<0.001)$ as highly significant.

\section{Observation}

A total of 150 eligible pediatric patients were included in this study and randomized into three different groups. As depicted in Table 1, the three groups were comparable in baseline characteristics as the difference did not reach statistical significance $(p>0.05)$. Patients' vital parameters were monitored for one hour following start of treatment. The three groups did not show a statistically significant change in parameters $(p>0.05)$ as shown in Table 2 . The primary outcome studied, which is about seizure recurrence at 24 hours, did not show a statistically significant difference in three groups ( $p>0.05)$. Also, seizure recurrence at 1 week did not reach a statistically significant difference. However, time to regain consciousness (TTRC) and seizure recurrence at 3 months were significantly

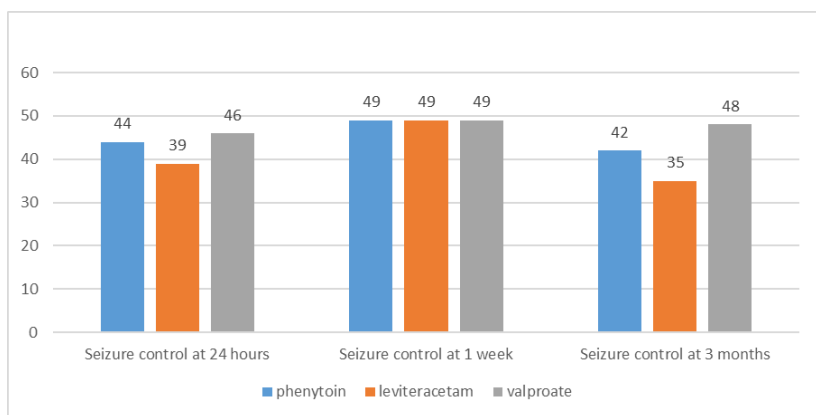

Figure 1. Comparison of seizure control at different time intervals. less in valproate group $(p<0.05)$. Results are summarized in Fig. 1 and Table 3. Subgroup analysis in focal and generalized seizures is shown in Table 4. A statistically significant control of seizures was seen in generalized seizures at 24 hours and 3 months in valproate group compared to levetiracetam $(p<0.05)$, but not to phenytoin. No significant difference was observed in focal seizure subgroups (p>0.05).

\section{Discussion}

Active seizures are usually controlled with shorter-acting agents like benzodiazepines. ${ }^{9,10}$ Longer-acting agents are started afterwards to augment seizure control and to prevent recurrence. The present study compares efficacy of IV phenytoin, IV levetiracetam and IV valproate for management of seizure in children. Diazepam was administered to control seizures. The patients were then randomly assigned to a study group and loaded with one of the anticonvulsants to be studied. All the three groups were comparable in age and sex distribution and in various anthropometric parameters like weight, height, head circumference and body mass index. We included only generalized and focal (simple and complex) seizures for comparison. The impact of different drugs on various cardiorespiratory parameters was studied and side-effects was, if any, recorded.

The primary outcome measure in our study was the cessation of seizure activity for 24 hours. At 24 hours seizures were controlled in 44 patients (88\%) out of 50 patients in phenytoin group, $39(78 \%)$

Table 3. Primary and secondary outcome measures in three groups

\begin{tabular}{lccc}
\hline \multirow{2}{*}{ Outcome measure } & \multicolumn{3}{c}{ Study group } \\
\cline { 2 - 4 } & Phenytoin $(n=50)$ & Levetiracetam $(n=50)$ & Valproate $(n=50)$ \\
\hline Seizure control at 24 hours & $44(88.0)$ & $39(76.0)$ & $46(92.0)$ \\
TTRC (minutes) & $122.34 \pm 45.41$ & $120.82 \pm 42.80$ & $75.04 \pm 30.66$ \\
Seizure control at 1 week & 49 & 49 & 49 \\
Seizure control at 3 months & 42 & 35 & 48 \\
\hline
\end{tabular}

Values are presented as mean \pm standard deviation or number (\%).

TTRC, time to regain consciousness.

Table 4. Subgroup analysis

\begin{tabular}{|c|c|c|c|c|c|c|}
\hline \multirow{3}{*}{ Outcome measure } & \multicolumn{6}{|c|}{ Study group } \\
\hline & \multicolumn{2}{|c|}{ Phenytoin } & \multicolumn{2}{|c|}{ Levetiracetam } & \multicolumn{2}{|c|}{ Valproate } \\
\hline & $F(n=13)$ & $G(n=37)$ & $F(n=13)$ & $G(n=37)$ & $F(n=11)$ & $G(n=39)$ \\
\hline Seizure control at 24 hours & 12 & 33 & 11 & 28 & 9 & 38 \\
\hline Seizure control at 3 months & 11 & 31 & 11 & 24 & 10 & 38 \\
\hline
\end{tabular}

F, focal; $G$, generalized. 
out of 50 patients in levetiracetam group and 46 (92\%) out of 50 patients in valproate group ( $p=0.115)$. The relative risk of seizure recurrence for levetiracetam and phenytoin groups when compared to valproate was 2.75 and 1.5 , respectively. This means valproate appears to be marginally better than the other two for acute seizure control even though the difference was not statistically significant. Misra et al. ${ }^{19}$ found that SE was aborted in $66 \%$ of patients with valproate, and in $42 \%$ of patients with phenytoin ( $p>0.05)$. In the study of Rai et al., ${ }^{20}$ seizures were controlled in $80 \%$ in valproate group and $92 \%$ in phenytoin group at 24 hours $(p=0.203)$. Goraya et al. ${ }^{21}$ and Abend et al. ${ }^{22}$ found similar results with intravenous levetiracetam. The mean time to regain consciousness in phenytoin, levetiracetam and valproate groups was $122.3 \pm 45.4,120.8 \pm 42.8$, and $75.0 \pm 30.7$ minutes (mean $\pm S D$ ), respectively. Patients in valproate group regained consciousness earlier than both phenytoin and levetiracetam group patients ( $p<0.0001)$. In the study Rai et al., ${ }^{20}$ patients initially received IV diazepam no difference in time taken to regain consciousness was observed between valproate and phenytoin groups. Yu et al. $^{23}$ found that the time taken for mental status recovery after IV valproate was less than 60 minutes in all patients with SE.

There was no significant difference in the three groups in various vital parameters like heart rate, systolic blood pressure, $\mathrm{SpO}_{2}$ and respiratory rate, which is consistent with the findings from other studies. ${ }^{23-25}$ Out of all patients in the three study groups only one patient from valproate group had a repeat seizure at the end of first week ( $p=0.372$ ). At 3 months follow-up, seven (14.28\%) out of 49 patients in phenytoin group, 14 (28.57\%) out of 49 in levetiracetam group and two (4\%) out of 50 patients in valproate group had a seizure recurrence $(p=0.0032)$. Valproate was significantly better for seizure control at 3 months than levetiracetam and the comparison between other groups revealed insignificant difference.

In our study most of the patients had generalized status, so we measured different outcome parameters in the generalized seizure category also. The number of patients with generalized status were 37 in phenytoin group, 37 in levetiracetam group and 39 in valproate group, out of total 50 patients in each group ( $p>0.05)$. Comparing seizure control at 24 hours in generalized seizures, valproate appeared better than levetiracetam ( $p=0.006)$, but comparable to phenytoin ( $p=0.194$ ). Further seizure control at 3 months in generalized seizures was better in valproate as compared to levetiracetam ( $p=0.0005)$ but similar to phenytoin ( $p=0.09$ ).

Adverse un-wanted side effects reported in our study were somnolence in two patients in levetiracetam group at 1 week follow-up. The age of both the patients was less than 3 months. The effects were not severe to warrant discontinuation of drugs. One patient at age of 11 months from valproate group had drug-induced hyperammonemia at 3 months follow-up requiring discontinuation of the drug. The limitations of study were that continuous EEG monitoring during acute phase was not possible and serum drug levels were not done in all patients at different intervals except those who had a seizure recurrence.

Valproate was better in providing long term seizure control, especially in generalized seizures, than levetiracetam and had a comparable effect to that of phenytoin. We also found that IV levetiracetam and IV valproate were comparable to IV phenytoin in terms of seizure control in acute setting. All the three are safe and efficacious. From the positive results in our study it can be concluded that levetiracetam and valproate can be recommended for inclusion in the treatment protocols of SE and as an initial loading dose in children requiring long term anticonvulsants. Although our experience with IV levetiracetam and IV valproate loading in pediatric seizure emergencies indicates that both are safe and effective, additional research is warranted to investigate further use of these drugs for treating seizure emergencies in pediatric patients. The present study compares efficacy of IV phenytoin, IV levetiracetam and IV valproate for seizure management in children and is, to the best of our knowledge, the only one available comparing these three drugs together.

\section{References}

1. McAbee GN, Wark JE. A practical approach to uncomplicated seizures in children. Am Fam Physician 2000;62:1109-16.

2. Vining EP. Pediatric seizures. Emerg Med Clin North Am 1994;12:973-88.

3. Singhi S, Singhi P, Dass R. Status epilepticus: emergency management. Indian J Pediatr 2003;70 Suppl 1:S17-22.

4. Hanhan UA, Fiallos MR, Orlowski JP. Status epilepticus. Pediatr Clin North Am 2001;48:683-94.

5. Treatment of convulsive status epilepticus. Recommendations of the epilepsy foundation of America's Working Group on status epilepticus. JAMA 1993;270:854-9.

6. Tullu MS, Mukhija V. Status epilepticus. In: Gupte S, ed. Recent Advances in Pediatrics. Special Volume 14. New Delhi: Jaypee Brothers, 2004;1531.

7. Berg AT, Shinnar S, Testa FM, et al. Status epilepticus after the initial diagnosis of epilepsy in children. Neurology 2004;63;1027-34.

8. Raj D, Gulati S, Lodha R. Status epilepticus. Indian J Pediatr 2011;78: 219-26.

9. Shearer P, Riviello J. Generalized convulsive status epilepticus in adults and children: treatment guidelines and protocols. Emerg Med Clin North 
Am 2011:29:51-64.

10. Mikati MA. Status epilepticus. In: Kliegman RM, Stanton BF, Schor NF, St. Geme JW, Behrman RE, eds. Nelson textbook of pediatrics. 19th ed. Philadelphia: Saunders, 2011;2013-7.

11. Haberlandt $E$, Sigl $S B$, Scholl-Buergi $S$, Karall $D$, Rauchenzauner $M$, Rostásy K. Levetiracetam in the treatment of two children with myoclonic status epilepticus. EurJ PaediatrNeurol 2009;13:546-9.

12. Alehan F, Ozcay F, Haberal M. The use of levetiracetam in a child with nonconvulsive status epilepticus. J Child Neurol 2008;23:331-3.

13. Weber P. Levetiracetam in nonconvulsive status epilepticus in a child with Angelman syndrome. J Child Neurol 2010;25:393-6.

14. Cilio MR, Bianchi $R$, Balestri $M$, et al. Intravenous levetiracetam terminates refractory status epilepticus in two patients with migrating partial seizures in infancy. Epilepsy Res 2009;86:66-71.

15. Kirmani BF, Crisp ED, Kayani S, Rajab H. Role of intravenous levetiracetam in acute seizure management of children. Pediatr Neuro/ 2009;41:37-9.

16. Gallentine WB, Hunnicutt AS, Husain AM. Levetiracetam in children with refractory status epilepticus. Epilepsy Behav 2009;14:215-8.

17. Chez MG, Hammer MS, Loeffel M, Nowinski C, Bagan BT. Clinical experience of three pediatric and one adult case of spike-and-wave status epilepticus treated with injectable valproic acid. J Child Neuro/ 1999;14: 239-42.
18. Uberall MA, Trollmann R, Wunsiedler U, Wenzel D. Intravenous valproate in pediatric epilepsy patients with refractory status epilepticus. Neurology 2000;54:2188-9.

19. Misra UK, Kalita J, Patel R. Sodium valproate vs phenytoin in status epilepticus: a pilot study. Neurology 2006;67:340-2.

20. Rai A, Aggarwal A, Mittal H, Sharma S. Comparative efficacy and safety of intravenous valproate and phenytoin in children. Pediatr Neurol 2011;45:300-4.

21. Goraya JS, Khurana DS, Valencia I, et al. Intravenous levetiracetam in children with epilepsy. Pediatr Neurol 2008;38:177-80.

22. Abend NS, Monk HM, Licht DJ, Dlugos DJ. Intravenous levetiracetam in critically ill children with status epilepticus or acute repetitive seizures. Pediatr Crit Care Med 2009;10:505-10.

23. Yu KT, Mills S, Thompson N, Cunanan C. Safety and efficacy of intravenous valproate in pediatric status epilepticus and acute repetitive seizures. Epilepsia 2003;44:724-6.

24. Birnbaum AK, Kriel RL, Norberg SK, et al. Rapid infusion of sodium valproate in acutely ill children. Pediatr Neurol 2003;28:300-3.

25. Ramael $\mathrm{S}$, Daoust $A$, Otoul $C$, et al. Levetiracetam intravenous infusion: a randomized, placebo-controlled safety and pharmacokinetic study. Epilepsia 2006;47:1128-35. 